Int. J. Contemp. Math. Sciences, Vol. 2, 2007, no. 28, 1367 - 1370

\title{
A Generalized Integration by Parts ${ }^{1}$
}

\author{
K. Hedayatian \\ Department of Mathematics \\ Shiraz University, Shiraz 71454, Iran \\ hedayati@shirazu.ac.ir
}

\begin{abstract}
In this article a generalization of integration by parts for the RiemannStieltjes integral is presented.
\end{abstract}

Mathematics Subject Classification: 26A42

\section{Introduction}

Fundamental Theorem of Calculus. Suppose $F$ is a real-valued function which is differentiable on $[\mathrm{a}, \mathrm{b}]$. If the derivative $F^{\prime}$ is Riemann integrable over $[\mathrm{a}, \mathrm{b}]$, then $\int_{a}^{b} F^{\prime}(x) d x=F(b)-F(a)$.

This theorem appears in every classical analysis book and is extended in some directions. For instance, when $F$ is right differentiable on $[a, b) \backslash D$, where $D$ is a countable subset of $[a, b]$, then under some extra conditions the theorem is valid. We may, also have the theorem whenever $F$ is a complex-valued function that is absolutely continuous on [a,b] see for example [5, P.311, 320]. See also [1]-[4], [6] and [7]. In this paper we bring some conditions under which the fundamental theorem of calculus and integration by parts are valid for the Riemann-Stieltjes integral.

\section{Main Results}

Definition 1. Let the real valued function $f$ be defined on [a,b]. Also, let $\varphi$ be an strictly increasing function on $[\mathrm{a}, \mathrm{b}]$. For any $x \in[a, b]$ we define

$$
D(f, \varphi)(x)=\lim _{t \rightarrow x} \frac{f(t)-f(x)}{\varphi(t)-\varphi(x)} .
$$

Moreover, we denote the Riemann-Stieltjes integral of $f$ with respect to $\varphi$ over $[\mathrm{a}, \mathrm{b}]$ by $\int_{a}^{b} f d \varphi$. If $\varphi(x)=x$ then $\int_{a}^{b} f d \varphi=\int_{a}^{b} f d x$ is the Riemann integral of $f$ over $[\mathrm{a}, \mathrm{b}]$. It can be easily seen that for functions $f$ and $g$ the following properties hold:

Property 1. $D(f g, \varphi)=D(f, \varphi) g+f D(g, \varphi)$.

Property 2. $D(f / g, \varphi)=\frac{D(f, \varphi) g-D(g, \varphi) f}{g^{2}} \quad g(x) \neq 0 \quad \forall x \in[a, b]$.

\footnotetext{
${ }^{1}$ Research partially supported by a grant (no. 85-GR-SC-27) from Shiraz University Council.
} 
Property 3. Suppose that $f$ is continuous on $[\mathrm{a}, \mathrm{b}]$; and $\varphi$ is an strictly in creasing continuous function defined on $[a, b]$. Furthermore, let $g$ be defined on an interval I which contains the range of $f$ and $g^{\prime}(f(x))$ exists on [a,b]. If $h(t)=g(f(t))$ then $D(h, \varphi)(x)$ exists and

$$
D(h, \varphi)(x)=g^{\prime}(f(x)) D(f, \varphi)(x) \text { for all } x \in[a, b] .
$$

Theorem 1. Let $\varphi$ be an strictly increasing continuous function on [a,b]. Suppose that $f$ is a Riemann-Stieltjes integrable function with respect to $\varphi$ on [a,b]. If there is a function $F$ on $[\mathrm{a}, \mathrm{b}]$ such that

$$
D(F, \varphi)(x)=f(x), \forall x \in[a, b]
$$

then

$$
\int_{a}^{b} f d \varphi=F(b)-F(a) .
$$

Proof. Let $P=\left\{a=x_{0}<x_{1}<\cdots<x_{n-1}<x_{n}=b\right\}$ be a partition of [a,b]. Fix the interval $\left[x_{i-1}, x_{i}\right]$ and put

$$
g(t)=\left(F\left(x_{i}\right)-F\left(x_{i-1}\right) \varphi(t)-\left(x_{i}-x_{i-1}\right) F(t), x_{i-1} \leq t \leq x_{i} .\right.
$$

Continuity of $\varphi$ implies that $F$ is continuous on $\left[x_{i-1}, x_{i}\right]$, and so is $g$. Also,

$$
D(g, \varphi)(t)=\left(F\left(x_{i}\right)-F\left(x_{i-1}\right)\right)-\left(x_{i}-x_{i-1}\right) f(t), \quad x_{i-1} \leq t \leq x_{i},
$$

and $g\left(x_{i}\right)=g\left(x_{i-1}\right)$. Suppose $g(t)>g\left(x_{i-1}\right)$ for some $t \in\left(x_{i-1}, x_{i}\right)$. Let $x$ be a point on $\left[x_{i-1}, x_{i}\right]$ at which $g$ attains its maximum. So $x \in\left(x_{i-1}, x_{i}\right)$ and

$$
\frac{g(t)-g(x)}{\varphi(t)-\varphi(x)} \geq 0 \quad x_{i-1}<t<x .
$$

Letting $t \rightarrow x$, we have $D(g, \varphi) \geq 0$. On the other hand,

$$
\frac{g(t)-g(x)}{\varphi(t)-\varphi(x)} \leq 0 \quad\left(x<t<x_{i}\right)
$$

which shows that $D(g, \varphi)(x) \leq 0$. Thus, $D(g, \varphi)(x)=0$. Similarly, if $g(t)<g\left(x_{i-1}\right)$ for some $t \in\left(x_{i-1}, x_{i}\right)$, assume that $x$ is a point in $\left[x_{i-1}, x_{i}\right]$ at which $g$ attains its minimum. So $x \in\left(x_{i-1}, x_{i}\right)$ and $D(g, \varphi)(x)=0$. Furthermore if $g$ is a constant function on $\left[x_{i-1}, x_{i}\right]$ then $D(g, \varphi)(x)=0$ for all $x$ in $(\mathrm{a}, \mathrm{b})$. Thus, we conclude that there is a point $t_{i} \in\left(x_{i-1}, x_{i}\right)$ so that

$$
F\left(x_{i}\right)-F\left(x_{i-1}\right)=\left(\varphi\left(x_{i}\right)-\varphi\left(x_{i-1}\right)\right) f\left(t_{i}\right) .
$$

Fix $\varepsilon>0$. Since $f$ is Riemann-Stieltjes integrable with respect to $\varphi$ on [a,b] we have

$$
\left|\sum_{i=1}^{n}\left(\varphi\left(x_{i}\right)-\varphi\left(x_{i-1}\right)\right) f\left(t_{i}\right)-\int_{a}^{b} f d \varphi\right|<\varepsilon,
$$


for some partition $\left\{x_{o}=a<x_{1}<\cdots<x_{n-1}<x_{n}=b\right\}$. Hence

$$
\int_{a}^{b} f d \varphi=\sum_{i=1}^{n} F\left(x_{i}\right)-F\left(x_{i-1}\right)=F(b)-F(a) . \square
$$

Corollary 1. If $\varphi$ is an strictly increasing continuous function on [a,b], and $\varphi(x) \neq$ $0 \forall x \in[a, b]$ then

$$
\int_{a}^{b} \frac{d \varphi}{\varphi}=(\ln |\varphi|)(b)-(\ln |\varphi|)(a) .
$$

Proof. Let $f(x)=|\varphi(x)|$ and $g(x)=\ln x$ in Property 3. Then $D(h, \varphi)(x)=$ $\frac{1}{|\varphi(x)|} D(|\varphi|, \varphi)(x)=\frac{1}{\varphi(x)}$ where $h(x)=\ln |\varphi(x)|$. Now, apply Theorem 1.

Corollary 2. If $\varphi$ is an strictly increasing continuous function on [a,b] such that $\varphi(x) \neq 0, \forall x \in[a, b]$, then

$$
\int_{a}^{b} \varphi^{n} d \varphi=\frac{\varphi^{n+1}(b)}{n+1}-\frac{\varphi^{n+1}(a)}{n+1}
$$

for any real number $n \neq 1$.

Proof. By the proof of Corollary 1 we have

$$
D\left(\ln \left|\varphi^{n}\right|, \varphi\right)=n D(\ln |\varphi|, \varphi)=\frac{n}{\varphi} .
$$

Now, using Property 3 for $f(x)=\varphi(x)^{n}$ and $g(x)=\ln |x|$ we have $D\left(\ln \left|\varphi^{n}\right|, \varphi\right)=$ $\frac{1}{\varphi^{n}} D\left(\varphi^{n}, \varphi\right)$, so $D\left(\varphi^{n}, \varphi\right)=n \varphi^{n-1}$. Therefore, $D\left(\frac{\varphi^{n+1}}{n+1}, \varphi\right)=\varphi^{n}, n \neq-1$. Thus the result holds by Theorem 1 .

Theorem 2(Generalized Integration by Parts). Let $\varphi$ be an strictly increasing continuous function on $[\mathrm{a}, \mathrm{b}]$. Suppose that $F$ and $G$ are functions defined on $[\mathrm{a}, \mathrm{b}]$ such that $D(F, \varphi)(x)$ and $D(G, \varphi)(x)$ exist for every $a \leq x \leq b$, and are RiemannStieltjes integrable with respect to $\varphi$ on $[\mathrm{a}, \mathrm{b}]$. Then

$$
\int_{a}^{b} F D(G, \varphi) d \varphi=F(b) G(b)-F(a) G(a)-\int_{a}^{b} G D(F, \varphi) d \varphi .
$$

Proof. Since $\varphi$ is continuous and $D(F, \varphi)$ and $D(G, \varphi)$ exist, we conclude that $F$ and $G$ are continuous on [a,b]. Therefore, $F D(G, \varphi)$ and $G D(F, \varphi)$ are RiemannStieltjes integrable with respect to $\varphi$. Put $H(x)=F(x) G(x)$. By Property 1 we have

$$
D(H, \varphi)=F D(G, \varphi)+G D(F, \varphi)
$$

and Theorem 1 implies that

$$
\int_{a}^{b} D(H, \varphi) d \varphi=H(b)-H(a) .
$$


So the result holds.

Theorem 3. Let $\varphi$ be a strictly increasing function on [a,b] and suppose that $f$ is Riemann-Stieltjes integrable with respect to $\varphi$ on $[\mathrm{a}, \mathrm{b}]$. For $a \leq x \leq b$, put $F(x)=\int_{a}^{x} f d \varphi$. Then

(a) If $f$ is continuous at $x_{0}$ of $[\mathrm{a}, \mathrm{b}]$, then

$$
D(F, \varphi)\left(x_{0}\right)=f\left(x_{0}\right)
$$

(b) If $\varphi$ is continuous on [a,b], then $F$ is continuous on [a,b].

Proof. (a) Given $\varepsilon>0$, choose $\delta>0$ such that

$$
\left|f(t)-f\left(x_{0}\right)\right|<\varepsilon
$$

if $\left|t-x_{0}\right|<\delta$, and $a \leq t \leq b$. Hence, if

$$
x_{0}-\delta<s \leq x_{0} \leq t<x_{0}+\delta \text { and }
$$

$a \leq s<t \leq b$ we have

$$
\left|\frac{F(t)-F(s)}{\varphi(t)-\varphi(s)}-f\left(x_{0}\right)\right|=\left|\frac{1}{\varphi(t)-\varphi(s)} \int_{s}^{t}[f(u)-f(x)] d \varphi\right|<\varepsilon
$$

It follows that $D(F, \varphi)\left(x_{0}\right)=f\left(x_{0}\right)$.

(b) Since $f$ is bounded suppose that

$$
|f(t)| \leq M \text { for } a \leq t \leq b \text {. If } a \leq x<y \leq b,
$$

then $|F(y)-F(x)|=\left|\int_{x}^{y} f d \varphi\right| \leq M\left|\int_{x}^{y} d \varphi\right|=M|\varphi(y)-\varphi(x)|$. Since $\varphi$ is uniformly continuous for every $\varepsilon>0$ we see that $|F(y)-F(x)|<\varepsilon$, when $|x-y|<\delta$ for some $\delta>0$. This proves uniformly continuity of $F$ on $[\mathrm{a}, \mathrm{b}] . \square$

\section{REFERENCES}

1. M. W. Botsko and R. Gosser, Stronger Version of the Fundamental Theorem of Calculus, The American Mathematical Monthly, 93(1986), 294-296.

2. M. W. Botsko, An Invitation to Real Analysis, $3^{\text {rd }}$ Edition, Eadmer Press, Greensburg, PA, 2004.

3. M. W. Bostsko, A Fundamental Theorem of Calculus that applies to all Riemann Integrable Functions, Mathematics Magazine, 64 (1991), 347-348.

4. J. J. Koliha, A Fundamental Theorem of Calculus for Lebesgue Integration, The American Mathematical Monthly, 113, No. 6, (2006) 551-554.

5. Karl R. Stromberg,An Introduction to Classical Real Analysis. California: Wadsworth \& Brooks, 1981.

6. C. Swartz, Even more on the Fundamental Theorem of Calculus, Proyecciones, 12, No. 2(1993) 129-135.

7. C. Swartz and B. S. Thomson, More on the Fundamental Theorem of Calculus, The American Mathematical Monthly, 95, No. 7 (1988) 644-648.

Received: February 21, 2007 